\title{
CARACTERIZAÇÃO ESPACIAL DA ATIVIDADE APÍCOLA NA MICRORREGIÃO DE CAMPOS DO JORDÃO, SÃO PAULO, BRASIL
}

\author{
SPATIAL CHARACTERIZATION OF BEEKEEPING IN THE MICRO REGION OF CAMPOS \\ DO JORDAO, SÃO PAULO, BRAZIL
}

\begin{abstract}
Bendini, J.N. ${ }^{1 *}$; Orsi, R.O. ${ }^{2}$; Bendini, H.N. ${ }^{3}$; Souza, D.C. ${ }^{4}$ e Salum, R.B. ${ }^{5}$
${ }^{1}$ Departamento de Zootecnia. Instituto de Ensino Superior Múltiplo. Maranhão. Timon. Maranhão. Brasil. *jbendini@gmail.com

${ }^{2}$ Faculdade de Medicina Veterinária e Zootecnia. Universidade Estadual Paulista (FMV/UNESP). Botucatu, São Paulo. Brasil.

${ }^{3}$ Programa de Pós Graduação em Sensoriamento Remoto. Instituto Nacional de Pesquisas Espaciais. INPE. São José dos Campos, São Paulo. Brasil.

${ }^{4}$ Departamento de Zootecnia. Centro de Ciências Agrárias. Universidade Federal do Piauí (DZO/CCA/UFPI). Piauí. Brasil.

${ }^{5}$ Programa de Pós Graduação em Geologia e Geoquímica. Universidade de Federal do Pará-UFPA. Belém, Pará. Brasil.
\end{abstract}

\section{PaLAVRAS CHAVE ADICIONAIS}

Sistema Informação Geográfica. Mel.

\section{RESUMO}

Objetivou-se gerar uma base de dados relacionada à distribuição espacial, de colmeias e produtividade dos apicultores regionais, por meio do Sistema de Informações Geográficas (SIG) utilizando-se o Sistema de Posicionamento Global. Para a construção do banco de informações foi utilizado o software de processamento de informação georreferenciada. A microrregião (4 municípios e 54 apicultores) mostra concentrações (bolsões) de apicultores e de colmeias em determinadas localidades. São necessários mais estudos sobre a flora apícola local para se inferir sobre saturação de recursos para as abelhas, já que não foram observadas diferenças de produção entre os apiários isolados ou inseridos nos bolsões. O SIG mostrou-se eficiente para o monitoramento da localização e procedência da produção regional e no planejamento da distribuição dos apiários e colmeias, podendo dessa forma contribuir para o melhor aproveitamento da flora local e melhoria da produtividade dos apiários.

\section{SUMMARY}

This study aimed to generate a database

\section{AdDitional KeYWORDS}

Geographic Information System Honey.

related to the spatial distribution of hives and productivity of regional producers, through the Geographic Information System (GIS) using the Global Positioning System. To build the database of geographical information (GIS) software for processing of geo-referenced information was used. The microregion (4 municipalities and 54 beekeepers) shows concentrations (pockets) of beekeepers and hives in certain locations. Further studies are needed on the characteristics of the local flora for bees to infer about possible saturation of floral resources, since no differences were observed between honey production in apiaries isolated or inserted into the pockets. The GIS proved to be an efficient tool for monitoring the location and origin of regional production, and for planning the distribution of hives and apiaries and so, contribute to a better use of flora and improvement of productivity of the apiaries.

\section{INTRODUÇÃO}

A microrregião de Campos do Jordão (Vale do Paraíba Paulista) inclui os municípios de Campos do Jordão, Santo An- 
tônio do Pinhal, São Bento do Sapucaí e Monteiro Lobato (os 3 primeiros transformados em Áreas de Proteção Ambientais).

A exploração apícola resultou numa produção de $21600 \mathrm{~kg}$ de mel em Campos do Jordão em 2003 (IBGE, 2003), porém, não existem até o momento, dados precisos relativos a um levantamento do setor apícola regional. A ausência de informações referentes ao desenvolvimento da atividade dificulta seu planejamento e crescimento. $\mathrm{O}$ conhecimento de onde e como a atividade se desenvolve, bem como, informações quanto aos produtores e a produção, são parâmetros importantes para o incremento da apicultura na microrregião.

O Sistema de Informação Geográfica (SIG) é o conjunto das informações georreferenciadas. A aplicação de sistemas computacionais trata os dados geográficos e elementos espaciais, oferecendo a visão de informações representadas com suas localizações geográficas (Aronoff, 1991). Assim, pode-se compreender que o SIG é uma ferramenta interdisciplinar capaz de inserir e integrar, numa única base de dados, os apiários da microrregião e suas respectivas características produtivas. Muller (1998) sugere o mapeamento de apiários para o desenvolvimento apícola para uma região localizada na Serra da Mantiqueira.

O trabalho teve como objetivo mapear os apiários dos 4 municípios da microrregião de Campos do Jordão, a partir da utilização do SIG, bem como, gerar uma base de dados relativa às características da produção de mel dos apicultores regionais representados em um mapa temático referente à cobertura vegetal do território.

\section{MATERIALEMÉTODOS}

O levantamento das posições geográficas dos apiários foi realizado utilizando-se o Sistema de Posicionamento Global, aparelho GPS Garmin Etrex. Para a construção do banco de informações geográficas (SIG) foi utilizado o software de Processamento de Informação Georreferenciada (SPRING), desenvolvido pelo Instituto Nacional de Pesquisas Espaciais (INPE). Os pontos coletados pelo GPS foram sistematizados em um banco de dados, contendo as informações obtidas por meio de entrevistas direcionadas aos apicultores.

Todas as análises estatísticas de produtividade foram previamente testadas quanto a sua distribuição, através do teste de Lilliefors uma vez que o teste de normalidades por homocedasticidade por ShapiroWilk foi $\mathrm{p}<0,05$, revelando que os dados não são de uma população distribuída normalmente. Após este procedimento, adotouse o método não paramétrico (KruskallWallis). Todas as análises estatísticas foram realizadas considerando-se um nível de significância mínimo de $5 \%$, por meio do software BioEstat 5.0.

\section{RESULTADOSEDISCUSSÃO}

A microrregião de Campos do Jordão apresenta, de acordo com o presente estudo, 54 apicultores. Foram contabilizadas 954 colmeias (modelo Langstroth) na microrregião de Campos do Jordão, o que representa $5,5 \%$ do total de colmeias do estado de São Paulo (Zara Filho, 2007).

A tabela I revela que a média de colmeias por apicultor é de aproximadamente 16,3. Diante desses dados, observa-se que cerca de $80 \%$ dos apicultores tem menos de 20 colmeias, o que torna evidente tratar-se de uma atividade desenvolvida no âmbito familiar por pequenos produtores.

A partir do georreferenciamento, observou-se que em todos os municípios existem grandes concentrações (bolsões) de apicultores e de colmeias instaladas em determinadas localidades. Admitiu-se, a partir de informações técnicas, a presença de mais de 100 colmeias compartilhando raios de 1,5 km de distância plana para a denominação de um bolsão de apiários. Dessa maneira, identificou-se 5 bolsões na microrregião de Campos do Jordão, que 
Tabela I. Apicultores (Apic) e colmeias (Colm) na microrregião de Campos do Jordão. (Beekeepers (Apic) and hives (Colm) in the microregion of Campos do Jordao).

\begin{tabular}{lccccc}
\hline Municípios & Apic, $\mathrm{n}^{\circ}$ & Apic, $\%$ & Colm, no & Colm, \% & Colm/Apic \\
\hline Santo Antônio do Pinhal & 11 & 20,37 & 254 & 26,62 & 23,1 \\
Monteiro Lobato & 17 & 31,48 & 270 & 28,30 & 15,9 \\
Campos do Jordão & 10 & 18,52 & 163 & 17,08 & 16,3 \\
São Bento do Sapucaí & 16 & 29,63 & 267 & 27,99 & 16,7 \\
Valores Totais & 54 & 100 & 954 & 100 & - \\
Médias por Municípios & 13,5 & - & 238,5 & - & 18,0 \\
\hline
\end{tabular}

podem representar um grande número de abelhas forrageando em um mesmo raio de distância, competindo pelos mesmos recursos florais.

Os municípios de São Bento do Sapucaí e Monteiro Lobato concentram o maior número de apiários (bolsões II-III e IV-V, respectivamente). Dessa maneira, em São Bento do Sapucaí, 85,25\% dos apicultores compartilham, aproximadamente, a área trabalhada por suas abelhas, sendo que nos bolsões II e III existem 149 e 106 colmeias. Já em Monteiro Lobato essa porcentagem é inferior $(64,7 \%)$, porém também representa a maioria dos apiários do município. Os bolsões IV e V contemplam 101 e 111 colmeias, respectivamente. Os apiários dos municípios de Santo Antônio do Pinhal e Campos do Jordão compartilham, aproximadamente, a área trabalhada pelas abelhas (214 colmeias) em um único bolsão (I).

Apesar da proximidade entre os apiários contidos nos bolsões, tal fato não in- fluenciou na produção de mel desses apiários, considerando o valor de $\mathrm{H}$ menor que o valor tabelado não havendo então diferença significativa ( $p>0,05)$.

Em relação à produtividade dos bolsões e dos grupos isolados, pode-se observar (figura1) a distribuição dos dados, evidenciando que nos bolsões III e IV os valores de produtividade estão concentrados em torno da mediana, enquanto que para os bolsões I e II, e para os apicultores isolados, mais de $25 \%$ dos dados estiveram acima da mediana, o que indica uma tendência em haver maior produtividade nesses grupos.

Segundo Bendini (2010), todos os apicultores do bolsão I participam da Associação de Produtores Rurais de Santo Antônio do Pinhal, APRUSAP. A organização dos pequenos produtores é um meio de garantir o acesso aos programas de desenvolvimento rural, que têm como objetivo o incremento do nível tecnológico, o que pode explicar relativo aumento da

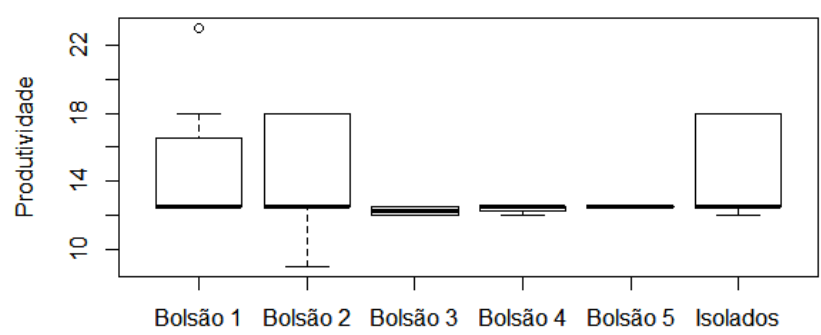

Figura 1. Boxplot das produtividades de mel dos apiários distribuídos nos bolsões $e$ isoladamente. (Box plot of the productivity of honey from apiaries distributed in pockets and singly). 
produtividade. Já os apicultores inseridos no bolsão II, segundo a mesma autora, apresentam suas colmeias instaladas em extensas áreas de bananicultura, o que para Santiago et al. (2006) pode ser considerado positivo para a apicultura já que a Musa paradisiaca não é mencionada na literatura como planta apícola, mas suas flores secretam néctar e podem ser exploradas para manter colônias de Apis mellifera durante o período de escassez de alimento.

A área trabalhada por qualquer colônia pode ser restringida por competição de coletoras de outras colmeias (Crane, 1983). Como não existem dados referentes ao número de enxames nidificados naturalmente na região, os resultados do presente estudo permitem inferir que os apicultores isolados demonstram certa tendência a apresentarem uma produtividade melífera superior aos demais apicultores contidos nos bolsões pelo fato dos mesmos não apresentarem suas abelhas compartilhando recursos florais com abelhas de outros apiários vizinhos. Estudos referentes à flora apícola da referida microrregião se fazem futuramente necessários para se atestar tal inferência.

A formação de bolsões de apiários durante o período estudado parece não inter-

\section{BIBLIOGRAFIA}

Aronoff, S. 1991. Geographic information systems: A management perspective. $2^{\text {nd }} \mathrm{ed}$. WDL Publications. Ottawa.

Bendini, J.N. 2010. Mel da microrregião de Campos do Jordão, estado de São Paulo: Uma proposta inicial para denominação de origem. Tese de Doutorado. Faculdade de Medicina Veterinária e Zootecnia. Universidade Estadual Paulista. Botucatu. $97 \mathrm{pp}$.

Crane, E. 1983. O livro do mel. $2^{\mathrm{a}}$ ed. Editora Nobel. São Paulo.

IBGE. Instituto Brasileiro de Geografia e Estatística. 2003. Pesquisa pecuária municipal, 2003. <http://www.ibge.gov.br/home/estatistica/ ferir na produtividade apícola em Campos do Jordão. Provavelmente as diferenças de produtividade relatadas pelos apicultores da referida microrregião se devam ao maior ou menor emprego de técnicas coerentes ao manejo adequado de suas colmeias. Bendini (2010) ao caracterizar a atividade desenvolvida pelos apicultores da região concluiu que embora os mesmos apresentem alguns avanços técnicos relacionados à garantia da qualidade do mel como, a utilização da tela excluidora e de materiais adequados à combustão do fumegador, não realizam a alimentação artificial em seus enxames durante o período de escassez de alimentos para as abelhas, o que pode acarretar numa baixa produtividade.

Conclui-se que as bases de dados aliadas ao mapa dos apiários resultantes da utilização do SIG representam importantes instrumentos para a caracterização do uso e cobertura da terra auxiliando no monitoramento da procedência do produto, no que tange à assistência técnica direcionada aos apicultores, principalmente para orientação quanto ao crescimento da apicultura microrregional e melhor distribuição de seus respectivos apiários, podendo surtir resultados positivos na produtividade melífera.

economia/ppm/2003/> (21/10/2006).

Muller, R.L. 1998. Dificuldades para um rápido desenvolvimento apícola no sul de Minas Gerais. Encontro Sobre Abelhas, 3. Ribeirão Preto. Anais... Universidade de São Paulo. São Paulo. Santiago, E. O., Sousa, A.M., Oliveira, R.N. 2006. A cultura da bananeira (Musa sp.) como fonte alternativa de alimento para manutenção de colônias de Apis mellifera durante o período seco na Caatinga. Congresso Brasileiro de Apicultura, 16. Anais... Aracaju.

Zara Filho, C. 2007. Panorama da apicultura no Estado de São Paulo em maio de 2007: diagnóstico agro-apicultura. SEBRAE-SP. São Paulo. 